\title{
Estudo da função simpático-adrenal em crianças submetidas a cirurgia cardíaca com hipotermia de superfície, perfusão limitada e parada circulatória
}

Richard K. FIRMIN*, Paul BOULOUX**, Paul ALLEN*, Ricardo de Carvalho LIMA*** J. C. LINCOLN*

RBCCV 44205-49

\begin{abstract}
FIRMIN, R. K.; BOULOUX, P.; ALLEN, P.; LIMA, R. C.; LINCOLN, J. C. - Estudo da função simpático-adrenal em crianças submetidas a cirurgia cardíaca com hipotermia de superfície, perfusão limitada e parada circulatória. Rev. Bras. Cir. Cardiovasc., 3(1): 55-59, 1988.

RESUMO: Os níveis de catecolamina plasmática foram medidos em 20 crianças (idade média de 6,00 $\pm 5,86$ meses; peso médio $5,3 \pm 1,82 \mathrm{~kg}$ ), durante a correção de defeitos cardiacos congênitos, usando-se a hipotermia de superfície $\left(26^{\circ} \mathrm{C}\right)$, perfusão cardiopulmonar limitada e parada circulatória $\left(15^{\circ} \mathrm{C}\right)$. Adrenalina e noradrenalina plasmática foram dosadas em amostras sangüineas arteriais seriadas, usando-se a cromatografia. A hipotermia de superfície produziu um significante aumento de ambas as catecolaminas. Durante o resfriamento central, os níveis caíram devido à hemodiluiçăo. Após o período de parada circulatória (23/64 minutos, média de 41,3), ocorreu um aumento das catecolaminas plasmáticas, que persistiu durante 0 reaquecimento. Após o reaquecimento, as catecolaminas plasmáticas permaneceram elevadas até o final do ato cirúrgico. Nossos resultados mostram que a técnica de hipotermia de superfície, perfusão cardiopulmonar limitada e parada circulatória, sob as nossas condiçōes de anestesia, produziu significante aumento da concentração de adrenalina e noradrenalina plasmática, porém o significado biológico é, ainda, inseguro.

DESCRITORES: função simpático-adrenal, crianças, cirurgia; hipotermia de supertície, crianças; perfusão limitada, crianças; parada circulatória, crianças.
\end{abstract}

\section{INTRODUÇĀO}

O uso, na infância, de hipotermia de superfície com perfusão cardiopulmonar limitada e parada circulatória tem-se tornado uma técnica bem padronizada, na correção de defeitos congênitos do coração. O grande impacto deste método, em termos de mortalidade imediata, levou a uma larga aplicação clínica sem, no entanto, ter sido precedida de uma detalhada avaliação experimental, a despeito das preocupaçōes referentes ao dano tecidual causado pela hipóxia.

A resposta simpático-adrenal em hipotermia de superfície com parada circulatória tem, recentemente, atraí- do o interesse de vários pesquisadores ${ }^{3,5,7}$. Estudamos um grupo de pacientes submetidos a cirurgia cardíaca, usando-se, de rotina, hipotermia com parada circulatória, na intenção de avaliar o grau do estresse simpáticoadrenal envolvido.

\section{CASUÍSTICA E MÉTODO}

Foram estudadas 20 crianças portadoras de defeitos congênitos cardiacos, as quais foram submetidas a cirurgia cardiaca corretiva. Idade, peso, superfície corpórea,

Trabalho realizado no Departamento de Cirurgia Cardiaca Pediátria do Bromptom Hospital. Londres, Inglaterra.

Aprovado para publicação em 10 de março de 1988.

- Do Bromptom Hospital.

*. Do St. Bartholomew's Hospital.

... Fellow em Cirurgia Cardiaca Pediátrica do Bromptom Hospital.

Endereço para separatas: Ricardo de Carvalho Lima. Av. Boa Viagem, 3160, Apto, 502. Boa Viagem. 50.000 Recife, PE, Brasil. 
FIRMIN, R. K.; BOULOUX, P.; ALLEN, P.; LIMA, R. C.; LINCOLN, J. C. - Estudo da função simpático-adrenal em crianças submetidas a cirurgia cardiaca com hipotermia de superfície, perfusão limitada e parada circulatória. Rev. Bras. Cir. Cardiovasc., 3(1): 55-59, 1988.

TABELA 1

\begin{tabular}{|c|c|c|c|c|}
\hline & VARIAÇĀO & MÉDIA & & $\begin{array}{l}\text { DESVIO } \\
\text { PADRAOO }\end{array}$ \\
\hline Idade & 2 dias - 18 meses & 6,0 & \pm & 5,86 meses \\
\hline Peso & $2,5-8,5 \mathrm{~kg}$ & 5,3 & \pm & $1,82 \mathrm{~kg}$ \\
\hline $\begin{array}{l}\text { Superficie } \\
\text { corpórea }\end{array}$ & $0,18-0,42 \mathrm{M}^{2} / \mathrm{SC}$ & 0,28 & \pm & $0,078 \mathrm{M}^{2} / \mathrm{SC}$ \\
\hline $\begin{array}{l}\text { Hemoglobina } \\
\text { Saturaçāo }\end{array}$ & $11,2-18,6 \mathrm{~g} / \mathrm{dl}$ & 15,2 & \pm & $2,21 \mathrm{~g} / \mathrm{dl}$ \\
\hline aórtica & $27-99 \%$ & 82,8 & \pm & $17,61 \%$ \\
\hline
\end{tabular}

TABELA 2

ETIOLOGIA

N.PACIENTES

Transposiçāo das Grandes Artérias

$$
\text { ( + PCA 2; + CIV 2) }
$$

Tétrade de Fallot

Drenagem Anômala Total das Veias Pulmonares

Comunicaçāo Interventricular (CIV)

Defeito do Septo Atrioventricular

Tronco Arterioso

Estenose Pulmonar

Estenose Aórtica, Estenose Mitral e Persistência do Canal Arterial (PCA)

\section{TOTAL}

saturação arterial e concentração de hemoglobina pré-operatórias encontram-se na Tabela 1 e os respectivos diagnósticos, na Tabela 2.

Foi utilizado um protocolo padronizado para anestesia, hipotermia de superfície, hipotermia central e reaquecimento (Figura 1).

A anestesia foi induzida com halotane, óxido nitroso, mistura de oxigênio e a manutençăo, com halotane associado a uma mistura de oxigênio suplementado com fentanil (1-2 mg/kg). Foram adicionados $5 \%$ de dióxido de carbono na mistura do gás inspirado, quando a temperatura nasofaríngea atingiu $30^{\circ} \mathrm{C}$.

Hipotermia de superfície com gelo foi utilizada para reduzir a temperatura nasofaríngea a $26^{\circ} \mathrm{C}$. A freqüência cardiaca foi anotada aos $34^{\circ} \mathrm{C}$ e, novamente, aos $26^{\circ} \mathrm{C}$, em 11 pacientes, ao mesmo tempo em que as amostras de catecolaminas foram tomadas. A esternotomia foi, então, realizada e a perfusão cardiopulmonar, estabelecida. Durante a perfusão, utilizaram-se oxigenadores Bentley, Irvine, Califórnia 92714 (BOS 5), com um perfusato de sangue (1 litro) mais eletrólitos. As crianças foram esfriadas a $15^{\circ} \mathrm{C}$ (nasofaríngeo) e a cirurgia corretiva foi, então, realizada durante um período de parada circu- latória total (10-67 minutos; média 40,4 $\pm 12,8 \mathrm{DP})$. Após a parada circulatória, as crianças foram reaquecidas até $33-37^{\circ} \mathrm{C}$, quando a perfusão foi interrompida.

Durante vários estágios do ato operatório, amostras de sangue heparinizado $(2 \mathrm{ml})$ foram retiradas através do catéter arterial utilizado para monitorização da pressāo arterial (Figura 1). As amostras foram colocadas em gelo $\left(4^{\circ} \mathrm{C}\right)$ até ao final da operação, quando foram centrifugadas e o plasma, separado. O plasma foi, então. rapidamente congelado a $-70^{\circ} \mathrm{C}$. A concentração de adrenalina plasmática foi medida usando-se cromatógrafo líqüido de alta pressão e detecção eletromecânica (valores normais: adrenalina $0,6 \mathrm{nmol} / \mathrm{L}$ e noradrenalina $0,6-2,0 \mathrm{nmol} / \mathrm{L}^{1}$.

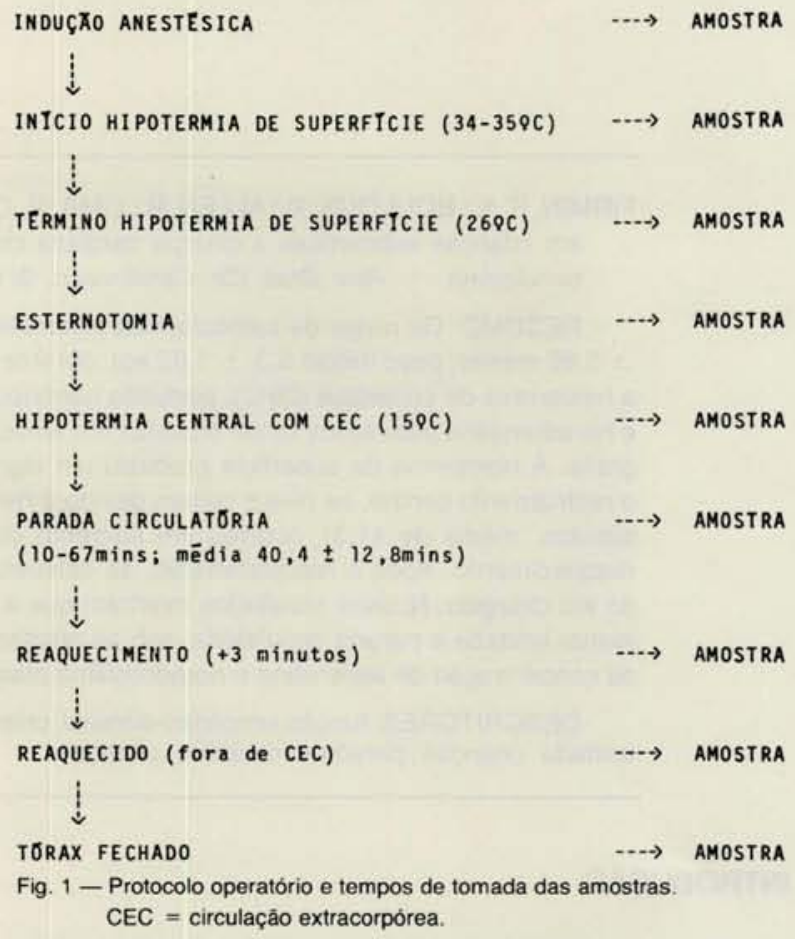

\section{ESTATÍSTICA}

Médias, desvio padrão e erro padrão da média foram calculados para o grupo de valores das catecolaminas e das variáveis dos pacientes. Comparaçāo entre os grupos de valores das catecolaminas foi feita usando-se a análise da variância. A influência das variáveis dos pacientes nos resultados foi analisada através de regressão múltipla. Regressão linear e variância foram usadas para comparar a resposta da freqüência cardiaca a $34^{\circ} \mathrm{C}$ e $26^{\circ} \mathrm{C}$; a regressão exponencial foi empregada para examinar a relaçāo entre tempo de parada e os niveis de catecolaminas pós parada. 
FIRMIN, R. K.; BOULOUX, P.; ALLEN, P.; LIMA, R. C.; LINCOLN, J. C. - Estudo da função simpático-adrenal em crianças submetidas a cirurgia cardiaca com hipotermia de superfície, perfusâo limitada e parada circulatória. Rev. Bras. Cir. Cardiovasc., 3(1) 55-59, 1988.

\section{RESULTADOS}

A hipotermia de superfície esteve associada a um significante aumento no plasma, da adrenalina ( $p<$ $0,005)$ e da noradrenalina ( $p<0,001)$ (Figura 2). Após o resfriamento central em perfusão, os niveis de ambas as catecolaminas caíram, refletindo a hemodiluição durante a perfusão. Após o período de parada circulatória, observou-se um variável aumento em ambas (adrenalina $p<0,1$ e noradrenalina $p<0,05$ ) (Figura 3). Estes aumentos estiveram melhor correlacionados com a duração da parada circulatória, como pode ser observado na equação de regressão exponencial (adrenalina $r=$ $0,65, p<0,001$ e noradrenalina $r=0,01$ ) (Figura 4). Os níveis de catecolaminas plasmáticas continuaram elevados após o reaquecimento, até o fechamento do tórax.

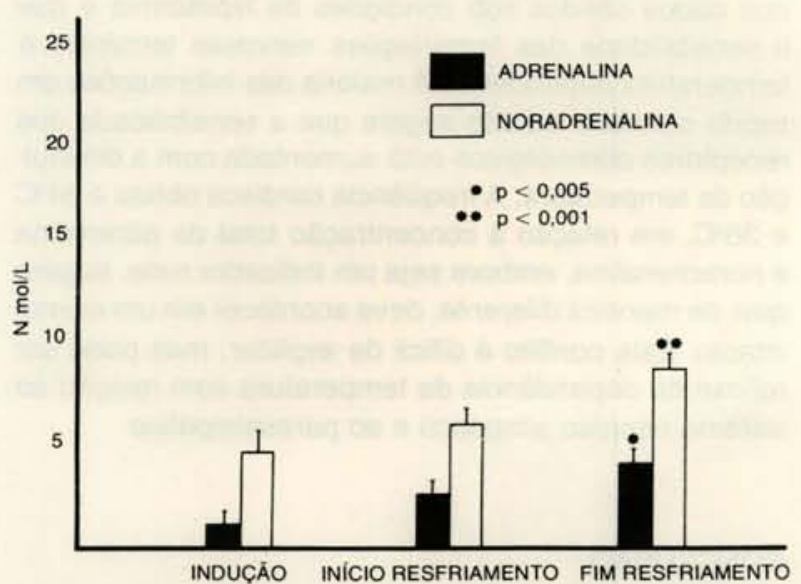

Fig. 2 - Efeitos da hipotermia de superficie nas concentraçōes das catecolaminas plasmáticas.

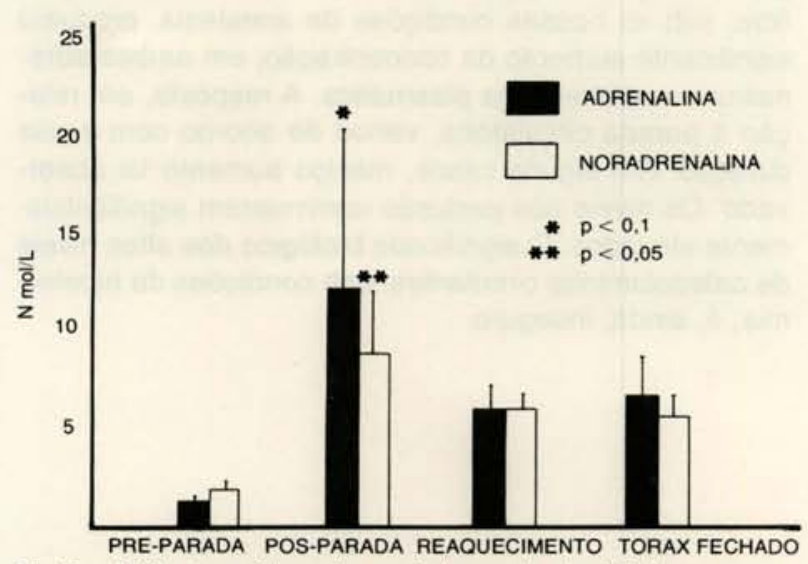

Fig. 3 - Efeito do resfriamento central, parada circulatória e reaquecimento nas concentraçōes de catecolaminas plasmáticas.

A resposta simpático-adrenal observada, nesses pacientes, não foi significativamente afetada por idade, peso, superfície corpórea, saturação arterial e concentração de hemoglobina.

A freqüência cardiaca apresentou um significante decréscimo na sensibilidade de sua resposta, relacionada com os valores combinados de adrenalina e noradrenalina a $26^{\circ} \mathrm{C}$, quando comparados aos $34^{\circ} \mathrm{C}(\mathrm{p}<$ 0,05 ) (Figura 5).
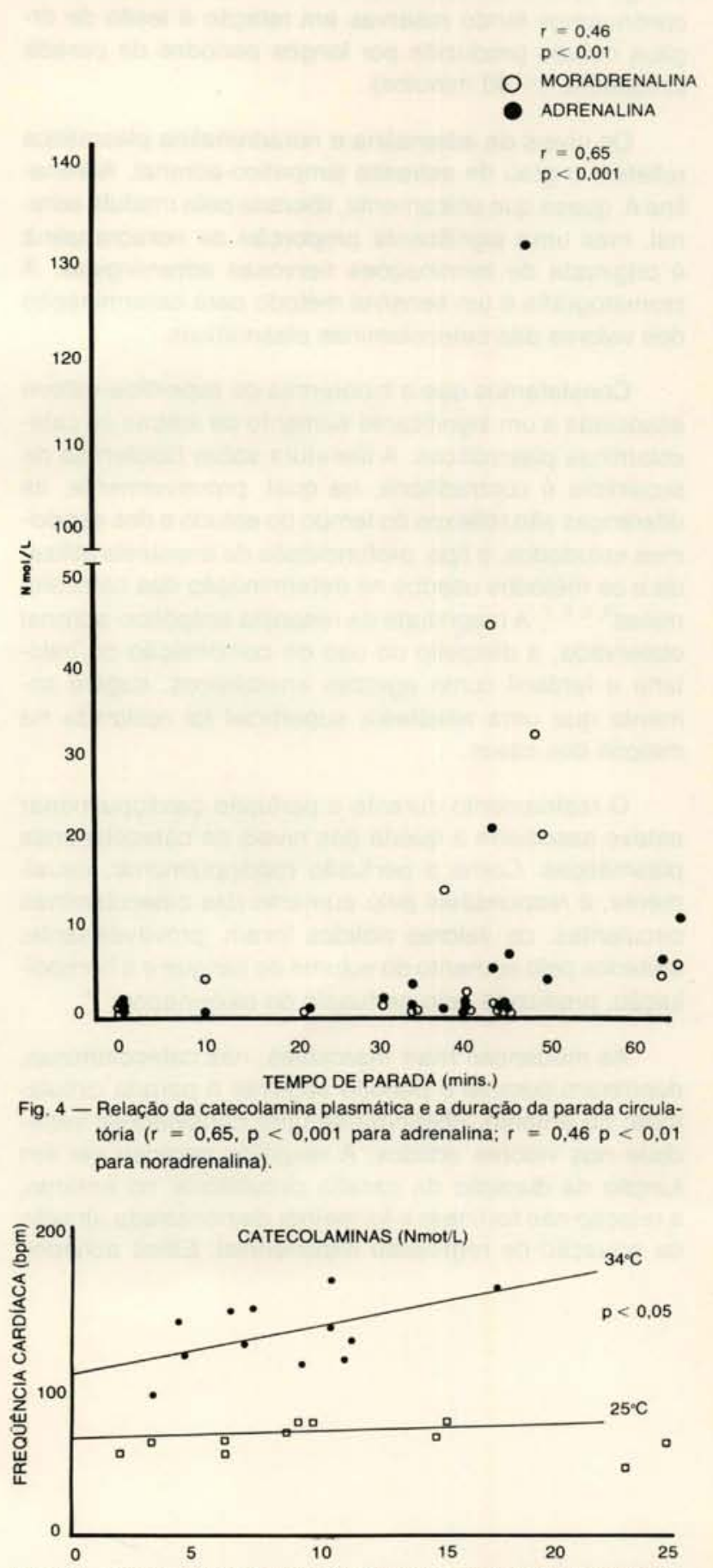

Fig. 5 - Relação entre a frequêencia cardiaca e a concentração plasmática total de adrenalina e noradrenalina. A diferença em inclinaçáo é significante. $(p<0,05)$. 
FIRMIN, R. K.; BOULOUX, P.; ALLEN, P.; LIMA, R. C.; LINCOLN, J. C. - Estudo da funçāo simpático-adrenal em crianças submetidas a cirurgia cardiaca com hipotermia de superficie, perfusão limitada e parada circulatória. Rev. Bras. Cir. Cardiovasc., 3(1): $55-59,1988$.

\section{DISCUSSĀO}

Empregamos a técnica de hipotermia de superfície com perfusão cardiopulmonar limitada e parada circulatória como método de escolha em cirurgia cardiaca aberta em crianças portadoras de defeitos congênitos com menos de $8 \mathrm{~kg}$ de peso. Embora nossos resultados, em geral, com esta técnica, tenham sido satisfatórios, continuamos tendo reservas em relação à lesão de órgãos nobres produzida por longos períodos de parada circulatória ( $>40$ minutos).

Os niveis de adrenalina e noradrenalina plasmática refletem o grau de estresse simpático-adrenal. Adrenalina é, quase que unicamente, liberada pela medula adrenal, mas uma significante proporção de noradrenalina é originada de terminaçōes nervosas adrenérgicas. A cromatografia é um sensivel método para determinaçāo dos valores das catecolaminas plasmáticas.

Constatamos que a hipotermia de superficie esteve associada a um significante aumento de ambas as catecolaminas plasmáticas. A literatura sobre hipotermia de superfície é contraditória, na qual, provavelmente, as diferenças são reflexos do tempo do estudo e dos espécimes estudados, o tipo, profundidade da anestesia utilizada e os métodos usados na determinação das catecolaminas $^{3,5,6,7}$. A magnitude da resposta simpático-adrenal observada, a despeito do uso da combinação do halotane e fentanil como agentes anestésicos, sugere somente que uma anestesia superficial foi realizada na maioria dos casos.

O resfriamento durante a perfusão cardiopulmonar esteve associado à queda dos niveis de catecolaminas plasmáticas. Como a perfusão cardiopulmonar, usualmente, é responsável pelo aumento das catecolaminas circulantes, os valores obtidos foram, provavelmente, afetados pelo aumento do volume de sangue e a hemodiluição, produzida pelo perfusato do oxigenador ${ }^{2} 4$.

As mudanças mais marcantes, nas catecolaminas, ocorreram durante o período seguinte à parada circulatória; no entanto, observou-se uma considerável variedade nos valores obtidos. A resposta pareceu ser em função da duração da parada circulatória; no entanto, a relação não foi linear e foi melhor demonstrada através da equação de regressão exponencial. Estes achados são compativeis com os publicados experimentalmente, utilizando-se cordeiros recém-nascidos, ou que se encontravam em fase de desenvolvimento ${ }^{3,7}$. No entanto, os valores que encontramos foram maiores do que os observados nos modelos experimentais, nos quais um maior tempo de parada circulatória foi utilizado. $O$ fato da curva de resposta com respeito à duração da parada circulatória não ser linear sugere que os mecanismos envolvidos são mais complexos do que somente a falha do mecanismo de clearance das catecolaminas durante a ausência de circulação.

As catecolaminas plasmáticas continuaram elevadas depois da perfusāo, até que o tórax fosse fechado. Os valores obtidos foram mais uniformes, durante esse período, e nenhum paciente apresentou valores excessivamente altos imediatamente após o período pós parada.

Um dos problemas para se conseguir significância nos dados obtidos sob condições de hipotermia é que a sensibilidade das terminaçōes nervosas terminais é temperatura dependente. A maioria das informaçōes em tecido cardíaco isolado sugere que a sensibilidade dos receptores adrenérgicos está aumentada com a diminuição da temperatura. A freqüência cardiaca obtida a $34^{\circ} \mathrm{C}$ e $36^{\circ} \mathrm{C}$, em relação à concentração total de adrenalina e noradrenalina, embora seja um indicador rude, sugere que, de maneira diferente, deve acontecer em um animal intacto. Este conflito é difícil de explicar, mas pode ser reflexo da dependência da temperatura com relação ao sistema nervoso simpático e ao parassimpático.

\section{CONCLUSÃO}

A técnica de hipotermia de superfície, perfusão cardiopulmonar limitada e parada circulatória produz uma grande resposta simpático-adrenal. Hipotermia de superfície, sob as nossas condições de anestesia, produziu significante aumento da concentração, em ambas adrenalina e noradrenalina plasmática. A resposta, em relação à parada circulatória, variou de acordo com a sua duração. Em alguns casos, maciço aumento foi observado. Os níveis pós perfusāo continuaram significativamente elevados. O significado biológico dos altos níveis de catecolaminas circulantes, sob condições de hipotermia, é, ainda, inseguro. 
FIRMIN, R. K.; BOULOUX, P.; ALLEN, P.; LIMA, R. C.; LINCOLN, J. C. - Estudo da função simpático-adrenal em crianças submetidas a cirurgia cardiaca com hipotermia de superficie, perfusão limitada e parada circulatória. Rev. Bras. Cir. Cardiovasc., 3(1): $55-59,1988$.

RBCCV $44205-49$

FIRMIN, R. K.; BOULOUX, P.; ALLEN, P.; LIMA, R. C.; LINCOLN, J. C. - Simpathoadrenal function during cardiac surgery in infants using the technique of surface cooling, limited cardiopulmonary bypass and circulatory arrest. Rev. Bras. Cir. Cardiovasc., 3(1): 55-59, 1988.

ABSTRACT: Plasma catecholamine levels were measured in 20 infants (mean age $6.0 \pm 5.86$ months; mean weigh $5.3 \pm 1.82 \mathrm{Kg}$ ), undergoing correction of congenital heart defects using surface cooling $\left(26^{\circ} \mathrm{C}\right)$, limited cardiopulmonary bypass and circulatory arrest $\left(15^{\circ} \mathrm{C}\right)$. Plasma adrenaline and noradrenaline were assayed in serial arterial blood samples using cromatography and electrochemical techniques. Surface cooling produced a significant rise in adrenaline and noradrenaline: the levels of both catecholamines fell, however. during core-cooling on cardiopulmonary bypass. Following the period of circulatory arrest (23/64 min, mean $41.3 \mathrm{~min}$ ), there was a further increase in plasma catecholamines, which persisted during rewarming. Following rewarming, plasma catecholamines remained elevated untill the end of the surgical procedure. Our data show the technique of surface cooling, limited cardiopulmonary bypass and circulatory arrest produces a major sympathoadrenal response. The biological significance of high levels of circulating catecholamines under hypothermic conditions is uncertain.

DESCRIPTORS: simpathoadrenal function, children, surgery; surface cooling, children; cardiopulmonary bypass, children; circulatory arrest, children.

\section{REFERÊNCIAS BIBLIOGRÁFICAS}

1 CAUSON, R. C.; BROWN, M. J.; BOULOUX, P. M.; PERRET, D. - Anatytical differences in measurement of plasma catecholamines. Clin. Chem., 29(4): 735-737, 1983.

2 HOAR, P. F.; STONE, J. G.; FALTAS, A. N.; BENDIXEN, H. H.; HEAD, R. J.; BERKOWITZ, B. A. - Hemodynamic and adrenergic response to anesthesia and operation for myocardial revascularization. J. Thorac. Cardiovasc. Surg., 80(2): 242-248, 1980.

3 MOHRI, H.; PITTS, C. L.; SANDS, M. P.; MANHAS, D. R.; DILLARD, D. H.; MERENDINO, K. A. - Effect of surface-induced hypothermia and circulatory occlusion on plasma catecholamines. Surgery, 72(4): 596-603, 1972.
4 STANLEY, T.H.; BERMAN, L.; GREEN, O.; ROBERTSON, D. - Plasma cathecolamine and cortison responses to fentanyl-oxygen anesthesia for coronary artery operation. Anesthesiology, 53(1): 250-253, 1980.

5 TURLEY, K.; ROIZEN, M.; VLAHAKES, G. J.; GRAHAM, B.; EBERT, P. A. - Catecolamine response to deep hypothermia and total circulatory arrest. Circulation, 62(2 Pt. 2): 175-179, 1980.

6 WARNER, W. A.; ANTON, A. H.; ANDERSEN, T. W. SWOFFORD, L. J. - Ventricular fibrilation and catecholamine responses during profound hipothermia in dogs. Anesthesiology, 33(1): 43-51, 1970.

7 WOOD, M.; SHAND, D. G.; WOOD, A. J. - The sympathetic response to profound hypothermia and circulatory arrest. Canad. Anesth. Soc. J., 27(2): 125-131, 1980. 